\title{
ECLIPSE MAPPING AT X-RAY WAVELENGTHS
}

\author{
J.H.M.M. SCHMITT \\ Max-Planck-Institut für extraterrestrische Physik \\ D-85740 Garching, Germany
}

\begin{abstract}
Stellar coronae cannot be spatially resolved, and yet stellar coronae are likely to exhibit a similar amount of structure as the solar corona. Currently, the only way to infer structure from spatially unresolved data is to look for rotational modulation of the X-ray emission or eclipses in the case of binary systems. I will discuss some of the observations obtained and critically review the methods used to infer structure from these data. Particular emphasis will be placed on the ill-conditioned nature of the inversion problem, that makes it next to impossible to infer the possibly three-dimensional structure of stellar coronae.
\end{abstract}

\section{Introduction}

The corona of the Sun is the only corona which can be spatially resolved with current X-ray telescopes. With the grazing incidence telescopes flown on Skylab, and more recently on $Y O H K O H$, an impressive record of the ever changing appearance of the solar X-ray corona has been obtained. Its most characteristic feature is the high degree of structure observed. The observable corona is far away from isotropy, one must not be misled by the usually published false-color images which - because of their logarithmic scaling - do not disclose the full dynamic range of the Sun's coronal X-ray emission. In order to illustrate this point I have constructed a cumulative frequency-flux diagram (cf., Fig. 1), showing the integrated X-ray flux for the full solar disk vs. integrated surface area (both normalised to unity); note that this diagram was obtained from the $Y O H K O H$ image published by Acton et al. (1992). As can be seen from Fig. 1, the solar corona is far away from being uniform, with $90 \%$ of the total X-ray flux coming from less than $1 \%$ of the total area. Looking then at the Sun from a stellar perspective, where one does not have the luxury of spatially resolved data, it is clear that the overall soft X-ray emission from the Sun is dominated 


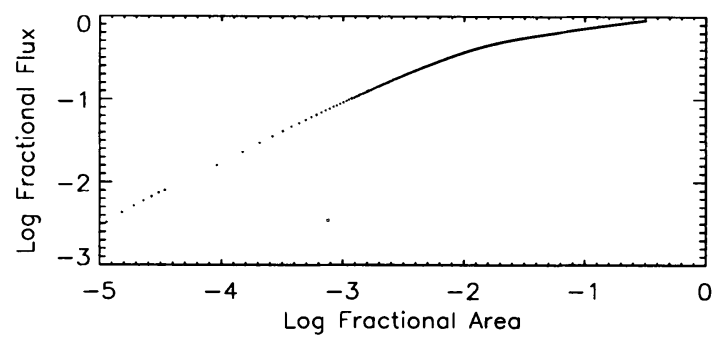

Figure 1. Normalised cumulative flux-area histogram for the Nov 12, $1991 Y O H K O H$ image of Sun. Note that most of the total flux is concentrated into rather small regions, so that the overall emission can be viewed as the summed emission from a few individual active regions.

by the summed contribution of a few active regions visible on the disk at any given time. The other components of the solar corona contribute only little to the total X-ray emission.

X-ray observations carried out with the Einstein and ROSAT Observatories have demonstrated the ubiquity of X-ray emission from stars in the HR-diagram. Except for cool giants of spectral type $\mathrm{K}$ and $\mathrm{M}$, all other types of cool stars are known to exhibit X-ray emission, thus indicating the presence of a corona. Specifically, Schmitt, Fleming and Giampapa (1995) and Schmitt (1996) have carried out X-ray surveys of volume-limited samples of nearby cool stars of spectral type F, G, K and M. In all cases one finds $\mathrm{X}$-ray detection rates close to $100 \%$, supporting the view that the formation of a corona represents a universal phenomemon, i.e., cool stars without a surrounding corona either do not exist or are at least very rare.

The X-ray luminosities of cool stars range from $\approx 310^{25} \mathrm{erg} / \mathrm{sec}$ for some of the lowest activity M-dwarfs to $>10^{32} \mathrm{erg} / \mathrm{sec}$ for the most active RS CVn and T Tauri stars. Even if one confines attention to stars of a specific type, one still finds a spread of 3 to 4 orders of magnitude in X-ray luminosity. The question then arises in what respect(s) stellar coronae must differ from the solar corona in order to accommodate the large X-ray outputs observed. Stellar coronae might occupy larger coronal volumes while the solar X-ray emission is usually confined to sizes scales much less than the pressure scale height. Stellar coronae might have larger filling factors the filling factor of the solar corona, obviously not a precisely defined quantity, must be small (cf., Fig. 1). And finally, the densities in stellar coronae might be higher which would also translate into larger emission measures since the plasma emissivity scales like the square of the density. All of these factors can contribute towards enhancing the total emission measure and we have no a priori way to determine which option Nature chooses. 


\section{How tó obtain structural information on stellar coronae?}

Obviously, the different coronal scenarios envisaged above differ in the ensuing spatial configuration of the X-ray emitting plasma. Unfortunately, given the spectral resolution of currently available $\mathrm{X}$-ray detectors, one is limited to essentially photometric data. In particular, it is impossible to disentangle - in the spectrum - the different components of binary systems, or to obtain line profiles; the only exception in this respect are the observations of the Fe XXI $1354 \AA$ line with the Hubble Space Telescope (cf., Robinson et al. 1995). Thus one is limited to the study of rotational modulation and eclipse mapping to infer structure information. As an aside we note that inference of structure can also be obtained - somewhat indirectly - from direct spectroscopic density measurements (cf., Schmitt, Haisch and Drake 1994).

Reconstruction methods, relying on photometric data alone, tend to lead to so-called ill-posed problems, i.e., problems whose solutions are not sufficiently constrained by the data. After all, in the case of photospheric starspot modelling one tries to infer a two-dimensional surface structure from a one-dimensional light curve. Stellar coronae may even be threedimensional entities, making the light curve inversion problem even more ill-posed.

A further difficulty for X-ray eclipse mapping lies in the large intrinsic variability of the observed X-ray emission. Most of the typically studied systems are so-called active stars which flare relatively frequently; light curve points affected by flaring must be removed, but this removal constitutes basically an ad hoc procedure. Little is known about the stability of stellar coronal X-ray emission over several rotation periods, while in contrast optical photometric light curves tend to remain stable over a few rotation periods.

Finally, the X-ray data have often relatively low signal to noise because of the low counting rates of the X-ray sources, and the data sampling is far inferior to that obtained by optical astronomers. Clearly, all of the above effects conspire against the task of eclipse mapping at X-ray wavelengths. Despite of all these problems, some progress has been made over the last few years, especially with observations obtained by the ROSAT satellite.

\section{Methods}

In the following section I briefly review methods that have been used to infer structural information from X-ray data. 


\subsection{CORONAL SPOT MODELLING}

Coronal spot modelling is an extension of the familiar spot modelling technique to X-ray data. In this approach one approximates the X-ray emitting features by simple geometrical shapes such as cylinders or conical sections. The number, positions, sizes and heights of these assumed features are varied until a best fit to the observed light curve is obtained. Such a method has been applied by Walter, Gibson and Basri (1983), White et al. (1990) and Ottmann, Schmitt and Kürster (1993) to the Einstein Observatory, EX$O S A T$ and ROSAT data of the eclipsing binary system AR Lac. Clearly, just like the case of photospheric spot modelling, one worries about the uniqueness of the light curve solutions and their sensitivity on the assumed geometric shapes.

\section{2. "WITHBROE-METHOD"}

By "Withbroe-Method" I denote the iterative scheme used by Siarkowski (1992) and collaborators for their light curve inversions. The technique was originally developed by Withbroe (1975) to derive solar differential emission distributions from spectral line intensities essentially in an intuitive fashion, and adapted by Siarkowski (1992) for the light curve inversion problem.

Denote by $f(x, y, z)$ the coronal intensity at the location $(x, y, z)$ and by $g_{i}(x, y, z)$ the so-called visibility function of the volume element located at $(x, y, z)$ at phase $\phi_{i} ; g_{i}(x, y, z)=1$ if the volume element under consideration is visible. The total expected intensity $I_{c a l c, i}$ observed at phase $\phi_{i}$ is then given by

$$
I_{\text {calc }, i}=\int d x d y d z \quad f(x, y, z) g_{i}(x, y, z) .
$$

If in the $\mathrm{n}^{\text {th }}$ iteration an estimate $f^{n}(x, y, z)$ is available, Siarkowski (1992) calculates an estimate $f^{n+1}(x, y, z)$ through

$$
f^{n+1}(x, y, z)=f^{n}(x, y, z) \frac{\sum_{i} \frac{I_{\text {observed }, i}}{I_{\text {calc }, i}} g_{i}(x, y, z)}{\sum_{i} g_{i}(x, y, z)} .
$$

In equation (2) $I_{\text {observed,i }}$ denotes the observed intensity at phase $\phi_{i}$, and $I_{c a l c, i}$ is evaluated by discretising equation (1). Equation (2) is iterated until $I_{\text {observed }, i}$ and $I_{\text {calc }, i}$ are identical in a statistical sense. The above formalism has been applied by Siarkowski (1992) to the EXOSAT data on AR Lac, and by Preś, Siarkowski and Sylwester (1995) to the EXOSAT data on TY Pyx. No mathematical justification for the use of equation (2) has been given. 


\subsection{INVERSION METHODS}

Equation (1), once written in vector form for all the observed phases $\phi_{i}$, is easily recognised as a Fredholm equation of the first kind, relating the observed intensities $I_{i}$ to the sought spatial distribution function $f(x, y, z)$. Denoting by $\mathbf{I}$ and $\mathbf{f}$ the vectors of the observed intensities and emissivities to be determined, and by $\mathbf{G}$ the matrix of visibility functions, we can "simply" write

$$
\mathbf{I}=\mathbf{G} \times \mathbf{f}
$$

and an abundance of methods exists to invert equation (3).

Following Lucy (1994), one should, however, not consider the light curve inversion problem as a problem in the solution of Fredholm equations but rather as a problem of parameter estimation. In X-ray astronomy, one is practically always dealing with counting experiments which can be easily formulated as maximum likelihood estimation problems. Let then $n_{i}$ denote the number of counts recorded in the time interval $\Delta t_{i}$ centered on phase $\phi_{i}$; further let the expected number of background events be $b_{i}$. Assuming then that the actual recorded integer number of counts $n_{i}$ is a Poissonian distributed random variable we can compute the overall probability of obtaining the observed data as

$$
\left.L=\log P=-\sum_{i}\left(\sum_{j} f_{j} g_{i j} \delta t_{i}\right)+b_{i}\right)+\sum_{i} n_{i} \log \left(\sum_{j} f_{j} g_{i j} \delta t_{i}+b_{i}\right),
$$

where we assume the function $f(x, y, z)$ discretised on some spatial grid denoted by an index $j$.

According to the principle of maximum likelihood one chooses the solution which maximises the system of equations

$$
\frac{\partial L}{\partial f_{k}}=-\sum_{i} g_{i k} \delta t_{i}+\sum_{i} n_{i} \frac{g_{i k} \delta t_{i}}{\sum_{j} f_{j} g_{i j} \delta t_{i}+b_{i}}=0 .
$$

Multiplying by $f_{k}$ transforms equation (5) into an fixed value equation which can be iteratively solved; its form is identical to that of equation (2), and therefore the "Withbroe-Method" is essentially an unregularised ML estimate.

\section{Comments}

\subsection{THE NEED FOR REGULARISATION}

The ML equations $(5)$ have the positivity constraint $\left(f_{k} \geq 0\right)$ implicitly incorporated. Obviously, if $f_{j}^{n}=0$, any subsequent iterates will also be zero, and none of the solution vector components can change sign if one starts 
out with an initial estimate satisfying the positivity constraint. Positivity is clearly a reasonable constraint to impose. We further note in this context that as long as one does not subtract background from the data (but rather models it as an additional known component), no negative signal can ever arise from a counting experiment. A further disadvantage of explicit background subtraction is that the statistics of the difference distribution becomes awkward because the difference between two Poisson distributions is not Poissonian (while the sum is).

As pointed out by Lucy (1994), as a consequence of the positivity constraint the "best fit" ML solutions tend to discretise; in other words, if a continuous distribution is used as input, the "best fit" output of a simulated experiment is not going to be a continous distribution but rather a solution with a few discrete components. This creates a problem for our eclipse mapping application since we must ensure that the structure we want to infer from our data is real and not introduced by imposing the positivity constraint.

One way to avoid the ill-conditioned nature of inversion problems is to impose a regularisation condition. The basic idea is to either use a priori knowledge or the principle of ignorance to force the solution to be as similar as possible to an "expected" one or to be as smooth as possible etc.. Mathematically this is achieved by adding to the likelihood the penality function $\alpha S$, where $\alpha$ is a regularisation parameter and $\mathrm{S}$ is given by

$$
S=\sum_{j}\left(f_{j}-\chi_{j}-f_{j} \ln \left(f_{j} / \chi_{j}\right)\right) .
$$

In equation (6) $\chi_{j}$ denotes the so-called default solution, which is often chosen to be a constant for maximum entropy reconstructions.

\subsection{REINVENTING THE WHEEL}

In the field of medicine, the technique of Positron Emission Tomography (PET; cf., Ter-Pogossian, Raichle and Sobel 1980) gives rises to mathematical problems very much related to that of eclipse mapping. In PET a radioactive nuclid (for example ${ }^{11} \mathrm{C}$ ) is administered to a patient; depending on the isotope chosen, different parts of tissue absorb different isotopes differently. The nuclids then decay via positron emission; in the body the positron annihilates very quickly with an electron, emitting - isotropically - two $511 \mathrm{keV}$ photons in opposite directions. With an array of $\gamma$-ray detectors such a photon pair can be detected by checking temporal coincidence. The probability of absorption of an $511 \mathrm{keV}$ photon in the tissue is very small, and therefore the technique is strictly linear in contrast to methods relying on - say - X-ray transmission. 
Mathematically the PET problem is equivalent to the X-ray eclipse mapping problem. In fact, the PET inversion problem is much better constrained since by arranging a circular array of $\gamma$-ray detectors around the tissue under study one effectively obtains emissivity measurements along all possible "line-of-sights" and hence a truly stereoscopic view. In the context of PET reconstruction techniques, Shepp and Vardi (1982) derived and applied equation (2), and placed it into a proper mathematical framework long before its usefulfulness was recognised for astronomical applications. Clearly, PET is much better constrained than X-ray eclipse mapping, in fact, it can be shown that a two-dimensional or three-dimensional object can be fully reconstructed by knowledge of its infinite set of all projections all all line of sights through the object. Thus, somewhat loosely speaking PET is more related to Doppler imaging rather than X-ray eclipsing mapping, although in Doppler imaging one is not really dealing with projection measurements.

\section{The case of $\alpha \mathrm{CrB} B$}

In essentially all cases of eclipsing binaries hitherto studied in X-rays both components of the binary systems are X-ray sources, which clearly adds to the difficulty of light curve inversion. The only exception to this is the binary system $\alpha \mathrm{CrB}$ (cf., Schmitt and Kürster 1993), which consists of an A-type star and a G-type star. The orbit inclination is close to $90^{\circ}$, therefore primary minimum ( $\mathrm{G}$ star in front of A star) is anular, while during secondary minimum the A star fully occults the $G$ star. The system is furthermore spectroscopic, and therefore the orbital parameters as well as stellar radii are known. Since A stars tend to be X-ray dark, one expects a total eclipse of the X-ray flux during optical secondary minimum. This is in fact observed (cf., Fig. 2), proving that first, the star $\alpha \mathrm{CrB}$ A is not an X-ray source, and demonstrating that the size of the $\mathrm{G}$ star corona is considerably smaller than the A-type star. The latter statement is in fact completely model-independent and can be quantified as follows: Denote by $r_{A}$ the radius of A star, and by $t_{e c l}$ the total eclipse duration; further assume that the apparent motion of the A star is in a straight line with speed $v_{A}$. The total area occulted (relative to the projected surface area of the A-type star, thus yielding an upper limit for the projected size of the $\mathrm{G}$ star corona) is given by $A_{o c c} / A_{A-s t a r}=(\phi-\sin (\phi)) / \pi$, where $\phi=\arccos \left(\frac{v_{A} t_{e c l}}{2 r_{A}}\right)$. The smaller length scale of this occulted area is $l_{o c c} / r_{A}=2-\frac{v_{A} t_{e c l}}{r_{A}}$ Putting in the actual numbers from the July $1992 \alpha \mathrm{CrB}$ observation one finds a total duration of $t_{e c l} \approx 16200 \mathrm{sec}$ for the totality of the X-ray eclipse, during which time the A-star moved by 1.3 radii. Thus the maximal projected size of the G-star corona can be 0.22 of the A-star's area, with a maximal size 


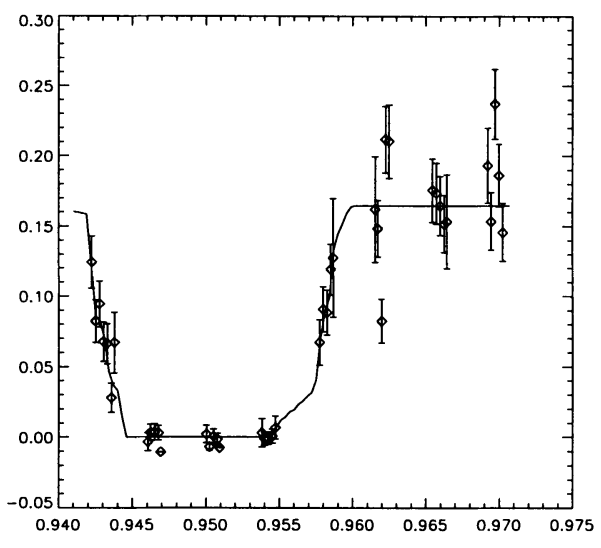

Figure 2. ROSAT PSPC light curve (in cts/sec) of $\alpha \mathrm{CrB}$ during secondary optical minimum on July 121992 vs. binary phase; note the totality of the X-ray eclipse while the A type star is in front of the G type star.

scale of $0.7 \mathrm{G}$-star radii.

More information on the distribution of the X-ray emission as projected onto the $\mathrm{G}$ star can be extracted from an analysis of the eclipse ingress and egress light curve. Applying the equations (5) and (6) to the observed PSPC light curve of $\alpha \mathrm{CrB}$, we can construct a series of solutions for various choices of the regularisation parameter $\alpha$. Since we are primarily interested in the question to what extent we can demonstrate the existence of structuring in the corona of $\alpha \mathrm{CrB} \mathrm{B}$, we choose a uniform solution as our default solution. For each choice of $\alpha$ and hence for each solution we can then compute a goodness of fit criterion describing how well the solution describes the observed light curve, for example in a $\chi^{2}$ sense. For small values of $\alpha$, the solution is unregularised, while for large values of $\alpha$ the solution tends towards the default solution, i.e., towards uniformity. These expectations are borne out by a plot (cf., Fig. 3), showing $\chi_{\text {red }}^{2}$ vs. $\alpha$; Fig. 3 shows that for values $\alpha \leq \sim 30$, the statistical quality of the solutions is basically constant, while larger values of $\alpha$ lead to solutions too uniform to be consistent with the observations.

In Fig. 4 and 5 we finally show two solutions for regularisation parameter choices of $\alpha=0$ and $\alpha=3$; the representation is chosen in such a way that the density of dots is proportional to the intensity emitted from each pixel. Comparing Fig. 4 and 5 it is obvious that increasing $\alpha$ tends to produce more uniform solutions. It is however also clear that most of the structure appearing in the unregularised solution (cf., Fig. 4) is also present in the regularised solution shown in Fig. 5, hence we argue that this structure is 


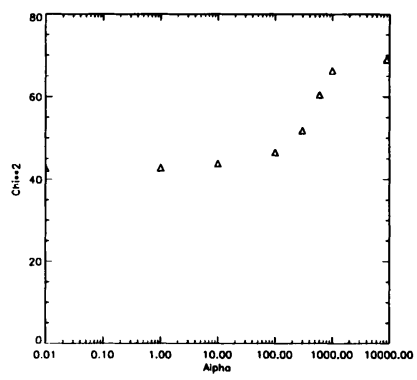

Figure 3. Goodness of fit $\chi^{2}$ vs. regularisation parameter $\alpha$ for the coronal image reconstructed from the ROSAT PSPC light curve of $\alpha \mathrm{CrB}$.
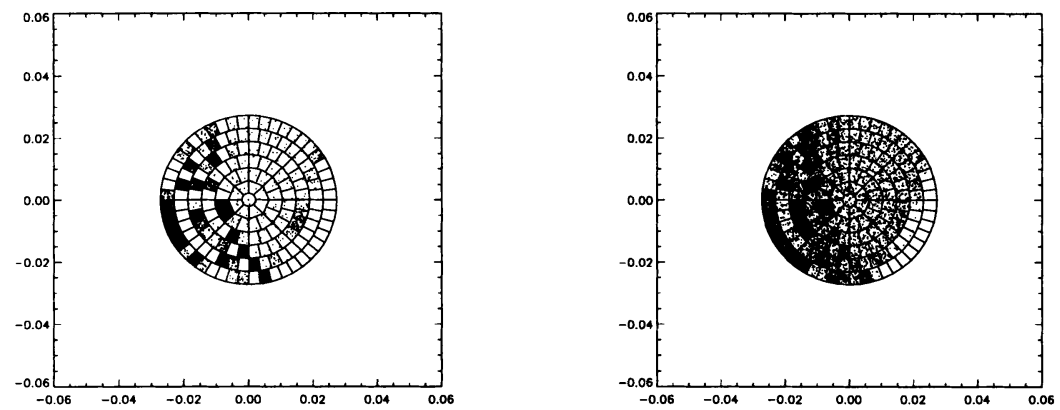

Figure 4. Left: Reconstructed corona image of $\alpha \operatorname{CrB~B~with~} \alpha=0$. Right: Same as Figure 4 , but $\alpha=3$.

unlikely to have been introduced by artefacts of the ML reconstruction.

\section{Conclusions}

In this review I have been able to cover only a small fraction of the relevant data pertaining to structural information at X-ray wavelengths. The basic issue whether the coronae of active stars are "large and tenous" or "small and compact" has still not been conclusively solved. However, compared to ten years ago when only Einstein Observatory and EXOSAT data were available, a much larger number of systems has been studied, and quite a few examples of rotational modulation and eclipses have been produced. While in the case of rotational modulation of a single star, the detection of a rotationally modulated $\mathrm{X}$-ray signal implies the existence of a maximum size scale (cf., Güdel 1996), in the case of an eclipsing binary, the X-ray emitting plasma can in principle still be far away from the stellar surfaces, and 
it is possible to interpret the $\mathrm{X}$-ray lightcurves as arising from "interbinary loops". However, it is essential to keep in mind that such reconstructions are not unique and light curve solutions of the same statistical quality can be obtained which locate all the emitting material near the stellar surfaces. From a physical point of view, the latter solutions appear to be more plausible, since first, the magnetic fields required for plasma confinement should be much larger near the stellar surfaces because of their extremely rapid decrease with distance, and second, in the context of "interbinary loops" there is little a priori reason why such loops should be concentrated in the orbit plane. Further, the a priori chances of detecting X-ray eclipses become smaller and smaller the further the $\mathrm{X}$-ray emitting plasma is moved away from the stellar surface; it thus appears strange that eclipses are seen in many of these systems. However, a final solution of the puzzle appears to be only possible once high spectral resolution data become available which will permit studying the densities of stellar coronae by investigating suitable line ratios and the dynamics of active stars by investigating the line profiles emitted by the coronal plasma.

\section{Acknowledgements}

The ROSAT project is supported by the German Bundesministerium für Bildung, Wissenschaft, Forschung und Technologie (BMBF/DARA) and the Max-Planck-Gesellschaft.

\section{References}

Acton, L., et al. (17 other authors), 1992, Science, Vol. 258, 618.

Güdel, M., 1996, this volume.

Lucy, L.B., 1994, A\&A , 289, 983.

Ottmann, R., Schmitt, J.H.M.M., and Kürster, M., 1993, ApJ. , 413, 710.

Preś, P., Siarkowski, M., and Sylwester, J., 1995, MNRAS, 275, 43.

Robinson, R.D., Airapetian, V.S., Maran, S.P. and Carpenter, K.G., 1995, IAU Symp. 176, Stellar Surface Structure, Poster Proceedings, ed. K. Strassmeier.

Schmitt J.H.M.M., \& Kürster M.: 1993, Science, Vol. 262, p. 215

Schmitt, J.H.M.M., Haisch, B.M., and Drake, J.J., 1994, Science, 265, 1420.

Schmitt, J.H.M.M., Fleming, T.A. and Giampapa, M.S., 1995, ApJ. in press.

Schmitt, J.H.M.M., 1996, in preparation.

Shepp, L.A:, and Vardi, Y., 1982, IEEE Transactions on Medical Imaging, Vol MI-1, 2, 113.

Siarkowski, M., 1992, MNRAS, 259, 453.

Ter-Pogossian, M.M., Raichle, M.E., and Sobel, B.E., 1980, Scientific American, 243, 170.

Walter, F. M., Gibson, D. M., and Basri, G. S. 1983, ApJ., 267, 665.

White, N. E., Shafer, R. A., Horne, K., Parmar, A. N., and Culhane, J. L., 1990, ApJ., $350,776$.

Withbroe, 1975, Solar Physics, 45, 301. 